\title{
Fluvial sediment pathways enlightened by OSL bleaching of river sediments and deltaic deposits
}

Supplementary File

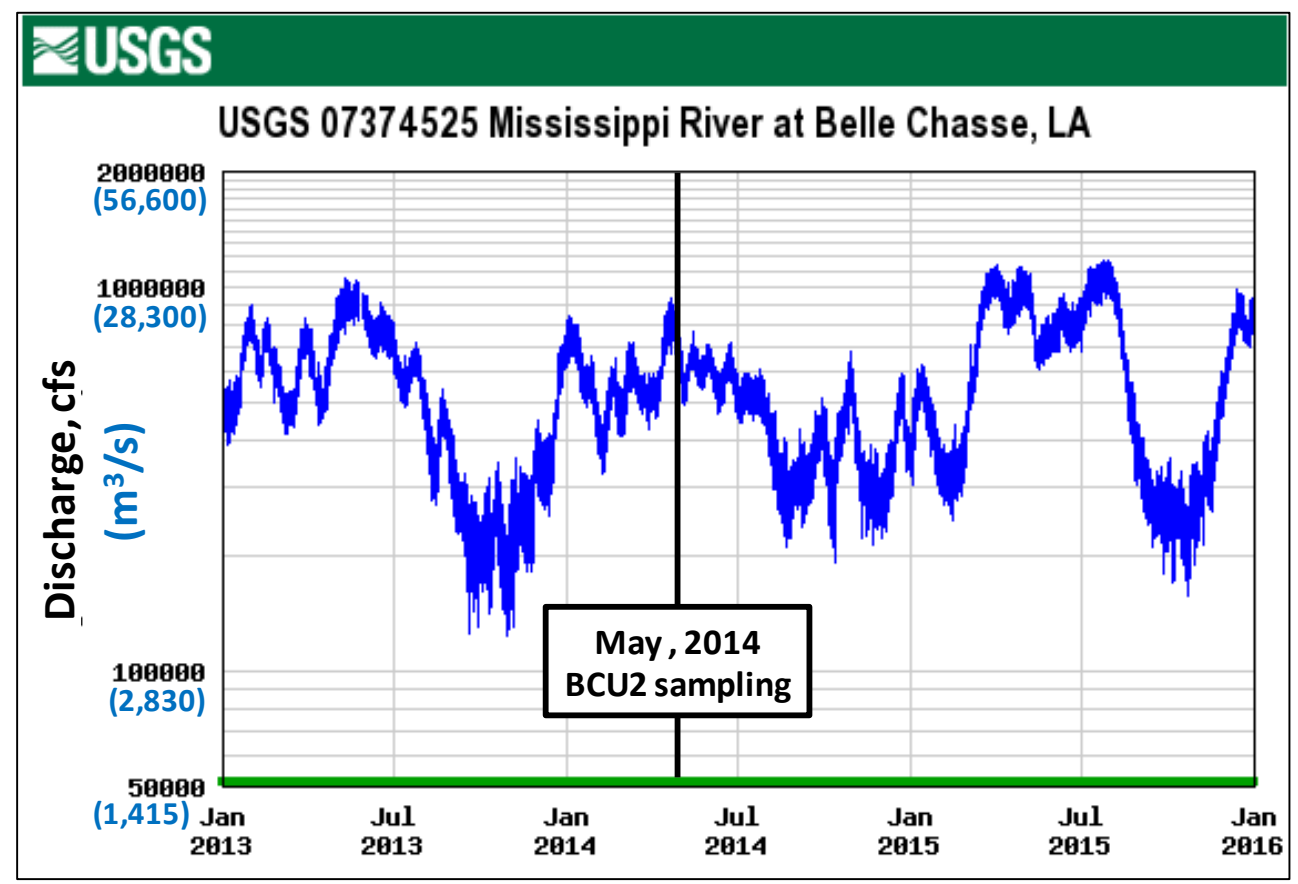

Figure S1. Mississippi River water discharge at Bell Chasse (river km 121), a monitoring station that generally represents discharge conditions at sample site BCU2, for a 3 year interval including the collection date of modern river bedload sand and suspended silt samples. 
Table S1. Minimum doses $\left(D_{e, b o t M A M}\right)$, central doses $\left(D_{e, C A M}\right)$, and residual doses for modern river sediments and sands isolated from sedimentary deposits, with (n) accepted aliquots ("al."). Dose rates are given for samples obtained from sedimentary deposits.

\begin{tabular}{|c|c|c|c|c|c|c|c|c|}
\hline $\begin{array}{l}\text { Sample } \\
\text { name }\end{array}$ & $\begin{array}{l}\text { Lab } \\
\text { code }\end{array}$ & $\begin{array}{l}\text { Grain } \\
\text { size } \\
(\mu \mathrm{m})\end{array}$ & $\begin{array}{l}\text { Al. } \\
\text { (n) }\end{array}$ & $\begin{array}{c}D_{e, \text { bootMAM }} \\
(\mathbf{G y})\end{array}$ & $\begin{array}{c}D_{e, C A M} \\
\text { Or } D_{e, M E A N} \\
(\mathbf{G y})\end{array}$ & $\begin{array}{c}\text { Residual dose } \\
\text { (Gy) }\end{array}$ & $\begin{array}{l}\text { Minimum } \\
\text { residual } \\
\text { dose }(G y)\end{array}$ & $\begin{array}{l}\text { Dose rate } \\
(\mathbf{G y} / \mathbf{k a})\end{array}$ \\
\hline \multicolumn{9}{|c|}{ Modern river suspended load } \\
\hline BCU2 I-1 & LV732 & $4-20$ & 4 & & $0.027 \pm 0.001^{\mathrm{a}}$ & $0.027 \pm 0.001$ & 0.026 & \\
\hline BCU2 I-2 & LV733 & $4-20$ & 2 & ------------- & $0.088 \pm 0.031^{\mathrm{a}}$ & $0.088 \pm 0.031$ & 0.057 & $\begin{array}{l}-------- \\
\end{array}$ \\
\hline BCU2 I-3 & LV734 & $4-20$ & 3 & & $0.040 \pm 0.014^{\mathrm{a}}$ & $0.040 \pm 0.014$ & 0.026 & \\
\hline BCU2 I-4 & LV735 & $4-20$ & 2 & +--- & $0.100 \pm 0.022^{\mathrm{a}}$ & $0.100 \pm 0.022$ & 0.078 & $-\cdots$ \\
\hline BCU2 I-5 & LV736 & $4-20$ & 2 & & $0.135 \pm 0.013^{\mathrm{a}}$ & $0.135 \pm 0.013$ & 0.122 & -- \\
\hline BCU2 I-3 & LV734 & $45-75$ & --- & $-\cdots$ & \multicolumn{3}{|c|}{ did not produce a measurable signal } & \\
\hline BCU2 I-5 & LV734 & $45-75$ & 4 & $-\ldots$ & $0.227 \pm 0.149^{\mathrm{a}}$ & $0.227 \pm 0.149$ & 0.078 & $-\cdots$ \\
\hline \multicolumn{9}{|c|}{ Modern river bedload } \\
\hline BCU2 I-6 & LV737 & $125-180$ & 36 & $0.027 \pm 0.051^{\mathrm{b}}$ & $1.617 \pm 0.288^{b}$ & $1.617 \pm 0.288$ & 1.329 & $\begin{array}{l}-------- \\
\end{array}$ \\
\hline BCU2 I-6 & LV737 & $180-250$ & 30 & $0.791 \pm 0.534^{\mathrm{b}}$ & $10.507 \pm 1.673^{b}$ & $10.507 \pm 1.673$ & 8.834 & $\begin{array}{l}-------- \\
\end{array}$ \\
\hline \multicolumn{9}{|c|}{ Lafourche mouth-bar deposits } \\
\hline GM I-2 & LV729 & $75-125$ & 64 & $1.992 \pm 0.082$ & $2.119 \pm 0.060$ & $0.127 \pm 0.104$ & 0.023 & $2.34 \pm 0.12$ \\
\hline BC I-1 & LV774 & $75-125$ & 68 & $2.936 \pm 0.291$ & $3.866 \pm 0.158$ & $0.930 \pm 0.338$ & 0.592 & $2.18 \pm 0.11$ \\
\hline CV I-1 & LV778 & $75-125$ & 84 & $2.686 \pm 0.124$ & $2.810 \pm 0.036$ & $0.124 \pm 0.132$ & -0.008 & $2.59 \pm 0.14$ \\
\hline CV II-1 & LV780 & $75-125$ & 82 & $2.525 \pm 0.096$ & $2.663 \pm 0.034$ & $0.138 \pm 0.104$ & 0.034 & $2.39 \pm 0.12$ \\
\hline RL I-1 & LV724 & $125-180$ & 54 & $2.794 \pm 0.225$ & $4.357 \pm 0.245$ & $1.563 \pm 0.339$ & 1.224 & $1.72 \pm 0.07$ \\
\hline RL I-2 & LV725 & $125-180$ & 58 & $2.897 \pm 0.246$ & $4.029 \pm 0.212$ & $1.132 \pm 0.331$ & 0.801 & $2.17 \pm 0.10$ \\
\hline FC I-2 & LV726 & $125-180$ & 29 & $1.386 \pm 0.101$ & $1.466 \pm 0.053$ & $0.080 \pm 0.116$ & -0.036 & $1.77 \pm 0.07$ \\
\hline FC I-1 & LV727 & $125-180$ & 48 & $1.084 \pm 0.214$ & $1.415 \pm 0.078$ & $0.331 \pm 0.232$ & 0.099 & $1.82 \pm 0.07$ \\
\hline GM I-1 & LV728 & $125-180$ & 40 & $2.000 \pm 0.092$ & $2.242 \pm 0.104$ & $0.242 \pm 0.142$ & 0.100 & $2.06 \pm 0.09$ \\
\hline LR I-1 & LV730 & $125-180$ & 54 & $2.548 \pm 0.121$ & $3.534 \pm 0.185$ & $0.986 \pm 0.225$ & 0.761 & $1.96 \pm 0.08$ \\
\hline LR I-2 & LV731 & $125-180$ & 47 & $2.697 \pm 0.290$ & $5.879 \pm 0.555$ & $3.182 \pm 0.639$ & 2.543 & $2.29 \pm 0.11$ \\
\hline SC I-1 & LV771 & $125-180$ & 30 & $3.408 \pm 0.241$ & $5.142 \pm 0.441$ & $1.734 \pm 0.513$ & 1.221 & $2.29 \pm 0.11$ \\
\hline BC I-2 & LV773 & $125-180$ & 53 & $3.320 \pm 0.164$ & $4.067 \pm 0.137$ & $0.747 \pm 0.218$ & 0.529 & $2.22 \pm 0.11$ \\
\hline CD I-2 & LV776 & $125-180$ & 59 & $2.044 \pm 0.066$ & $2.091 \pm 0.042$ & $0.047 \pm 0.080$ & -0.033 & $2.17 \pm 0.10$ \\
\hline CD I-1 & LV777 & $125-180$ & 71 & $1.808 \pm 0.126$ & $2.018 \pm 0.043$ & $0.210 \pm 0.135$ & 0.075 & $2.00 \pm 0.08$ \\
\hline DL I-2 & LV800 & $125-180$ & 69 & $2.337 \pm 0.064$ & $2.355 \pm 0.032$ & $0.019 \pm 0.073$ & -0.054 & $2.18 \pm 0.10$ \\
\hline DL I-1 & LV801 & $125-180$ & 72 & $2.371 \pm 0.093$ & $2.420 \pm 0.034$ & $0.049 \pm 0.101$ & -0.052 & $2.29 \pm 0.12$ \\
\hline
\end{tabular}


Table S1. Continued.

\begin{tabular}{|c|c|c|c|c|c|c|c|c|}
\hline \multicolumn{9}{|c|}{ Lafourche overbank deposits } \\
\hline EF II-2 & LV598 & $75-125$ & 11 & $2.759 \pm 0.140$ & $2.729 \pm 0.213$ & $-0.030 \pm 0.260$ & -0.290 & $3.01 \pm 0.19$ \\
\hline EF II-3 & LV599 & $75-125$ & 25 & $3.222 \pm 0.115$ & $3.279 \pm 0.082$ & $0.057 \pm 0.144$ & -0.087 & $2.41 \pm 0.13$ \\
\hline NV II-4a & LV653 & $75-125$ & 37 & $2.545 \pm 0.155$ & $2.821 \pm 0.129$ & $0.276 \pm 0.205$ & 0.071 & $2.39 \pm 0.13$ \\
\hline NV X-3 & LV471 & $75-125$ & 27 & $3.640 \pm 0.146$ & $4.800 \pm 0.338$ & $1.160 \pm 0.376$ & 0.784 & $3.13 \pm 0.18$ \\
\hline EF II-1 & LV596 & $75-125$ & 13 & $1.913 \pm 0.227$ & $1.979 \pm 0.118$ & $0.065 \pm 0.261$ & -0.196 & $3.03 \pm 0.20$ \\
\hline EF II-6 & LV721 & $75-125$ & 34 & $2.807 \pm 0.077$ & $2.815 \pm 0.067$ & $0.009 \pm 0.104$ & -0.095 & $2.44 \pm 0.13$ \\
\hline EF III-1a & LV654 & $75-125$ & 51 & $2.579 \pm 0.234$ & $3.656 \pm 0.158$ & $1.076 \pm 0.288$ & 0.788 & $2.63 \pm 0.15$ \\
\hline NV II-2 & LV641 & $90-180$ & 51 & $2.746 \pm 0.109$ & $2.794 \pm 0.071$ & $0.048 \pm 0.133$ & -0.085 & $2.41 \pm 0.13$ \\
\hline NV II-3 & LV642 & $90-180$ & 50 & $2.626 \pm 0.079$ & $2.642 \pm 0.067$ & $0.016 \pm 0.105$ & -0.089 & $2.49 \pm 0.14$ \\
\hline PV I-7 & LV587 & $90-180$ & 34 & $2.310 \pm 0.048$ & $2.321 \pm 0.049$ & $0.011 \pm 0.070$ & -0.059 & $2.50 \pm 0.13$ \\
\hline PV I-8 & LV646 & $90-180$ & 31 & $3.406 \pm 0.197$ & $5.020 \pm 0.356$ & $1.614 \pm 0.415$ & 1.199 & $2.54 \pm 0.15$ \\
\hline NV VIII-1 & LV416 & $75-180$ & 51 & $1.897 \pm 0.068$ & $1.967 \pm 0.054$ & $0.070 \pm 0.089$ & -0.019 & $2.72 \pm 0.18$ \\
\hline NV X-1 & LV419 & $75-180$ & 53 & $3.821 \pm 0.185$ & $4.230 \pm 0.140$ & $0.478 \pm 0.237$ & 0.241 & $2.49 \pm 0.14$ \\
\hline NV III-1 & LV432 & $75-180$ & 47 & $1.689 \pm 0.127$ & $1.912 \pm 0.066$ & $0.223 \pm 0.145$ & 0.078 & $2.60 \pm 0.16$ \\
\hline NV III-3 & LV433 & $75-180$ & 56 & $2.678 \pm 0.057$ & $2.684 \pm 0.047$ & $0.006 \pm 0.075$ & -0.069 & $2.37 \pm 0.13$ \\
\hline NV IV-1 & LV427 & $75-180$ & 31 & $1.780 \pm 0.214$ & $2.110 \pm 0.101$ & $0.330 \pm 0.241$ & 0.089 & $2.53 \pm 0.16$ \\
\hline NV-IV-2 & LV428 & $75-180$ & 61 & $2.044 \pm 0.112$ & $2.126 \pm 0.044$ & $0.082 \pm 0.123$ & -0.041 & $2.63 \pm 0.17$ \\
\hline NV V-1 & LV435 & $75-180$ & 44 & $2.392 \pm 0.152$ & $3.063 \pm 0.141$ & $0.671 \pm 0.212$ & 0.459 & $3.42 \pm 0.30$ \\
\hline NV V-2 & LV436 & $75-180$ & 42 & $2.389 \pm 0.090$ & $2.893 \pm 0.112$ & $0.504 \pm 0.146$ & 0.358 & $2.63 \pm 0.16$ \\
\hline NV VII-1 & LV415 & $75-180$ & 49 & $2.230 \pm 0.082$ & $2.282 \pm 0.065$ & $0.053 \pm 0.107$ & -0.054 & $2.69 \pm 0.17$ \\
\hline NV IX-1 & LV418 & $75-180$ & 68 & $2.381 \pm 0.113$ & $2.543 \pm 0.065$ & $0.161 \pm 0.133$ & 0.028 & $2.45 \pm 0.14$ \\
\hline PV I-4 & LV287 & $100-200$ & 31 & $2.470 \pm 0.249$ & $3.145 \pm 0.146$ & $0.675 \pm 0.294$ & 0.381 & $2.50 \pm 0.22$ \\
\hline PV I-5 & LV288 & $100-200$ & 45 & $2.947 \pm 0.245$ & $3.517 \pm 0.120$ & $0.570 \pm 0.278$ & 0.292 & $2.75 \pm 0.18$ \\
\hline
\end{tabular}


Table S2. Details of samples used for the paired sand and silt comparison. See Table S1 for sand dose rates and equivalent doses.

\begin{tabular}{|l|l|c|c|c|c|c|}
\hline \multicolumn{1}{|c|}{$\begin{array}{c}\text { Sample } \\
\text { name }\end{array}$} & $\begin{array}{c}\text { Lab } \\
\text { code }\end{array}$ & $\begin{array}{c}\text { Silt } \boldsymbol{D}_{\boldsymbol{e}, \boldsymbol{C A M}} \\
(\mathbf{G y})\end{array}$ & $\begin{array}{c}\text { Silt dose rate } \\
(\mathbf{G y} / \mathbf{k a})\end{array}$ & $\begin{array}{c}\text { Silt age } \\
(\mathbf{k a})\end{array}$ & $\begin{array}{c}\text { Sand } \\
\text { bootMAM age } \\
(\mathbf{k a})\end{array}$ & $\begin{array}{c}\text { Sand } \\
\text { CAM age } \\
(\mathbf{k a})\end{array}$ \\
\hline PV I-4 & LV287 & $4.013 \pm 0.223$ & $2.784 \pm 0.130$ & $1.441 \pm 0.104$ & $0.989 \pm 0.132$ & $1.259 \pm 0.125$ \\
\hline PV I-5 & LV288 & $5.144 \pm 0.132$ & $3.110 \pm 0.156$ & $1.654 \pm 0.093$ & $1.071 \pm 0.114$ & $1.278 \pm 0.096$ \\
\hline NV II-2 & LV641 & $2.834 \pm 1.114$ & $2.655 \pm 0.112$ & $1.067 \pm 0.422$ & $1.141 \pm 0.078$ & $1.161 \pm 0.072$ \\
\hline NV II-3 & LV642 & $3.108 \pm 0.028$ & $2.804 \pm 0.125$ & $1.109 \pm 0.051$ & $1.056 \pm 0.067$ & $1.062 \pm 0.065$ \\
\hline NV VIII-1 & LV416 & $1.952 \pm 0.025$ & $3.053 \pm 0.143$ & $0.640 \pm 0.031$ & $0.697 \pm 0.051$ & $0.722 \pm 0.051$ \\
\hline NV X-1 & LV419 & $3.729 \pm 0.052$ & $2.796 \pm 0.119$ & $1.334 \pm 0.060$ & $1.533 \pm 0.114$ & $1.725 \pm 0.113$ \\
\hline EF II-2 & LV598 & $2.500 \pm 0.049$ & $3.411 \pm 0.153$ & $0.733 \pm 0.036$ & $0.918 \pm 0.075$ & $0.908 \pm 0.091$ \\
\hline EF II-3 & LV599 & $3.712 \pm 0.061$ & $2.720 \pm 0.109$ & $1.365 \pm 0.059$ & $1.335 \pm 0.085$ & $1.359 \pm 0.079$ \\
\hline
\end{tabular}

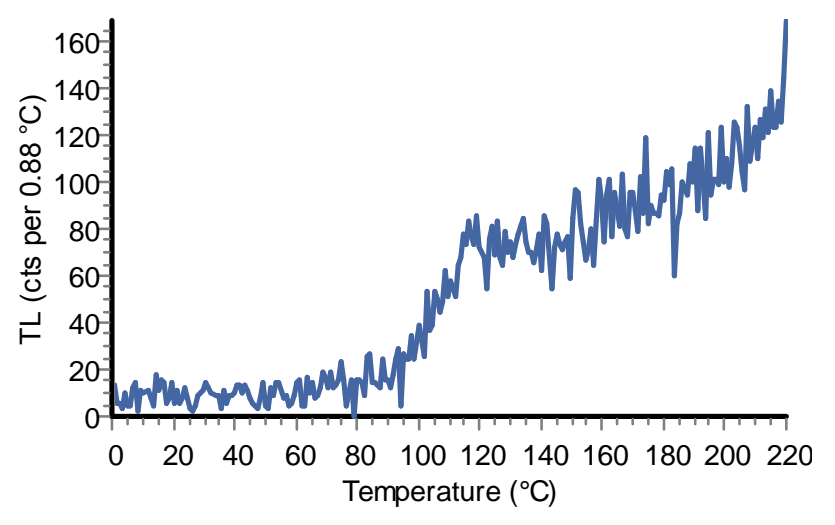

Figure S2. Typical TL response for PV I-4 silt. 


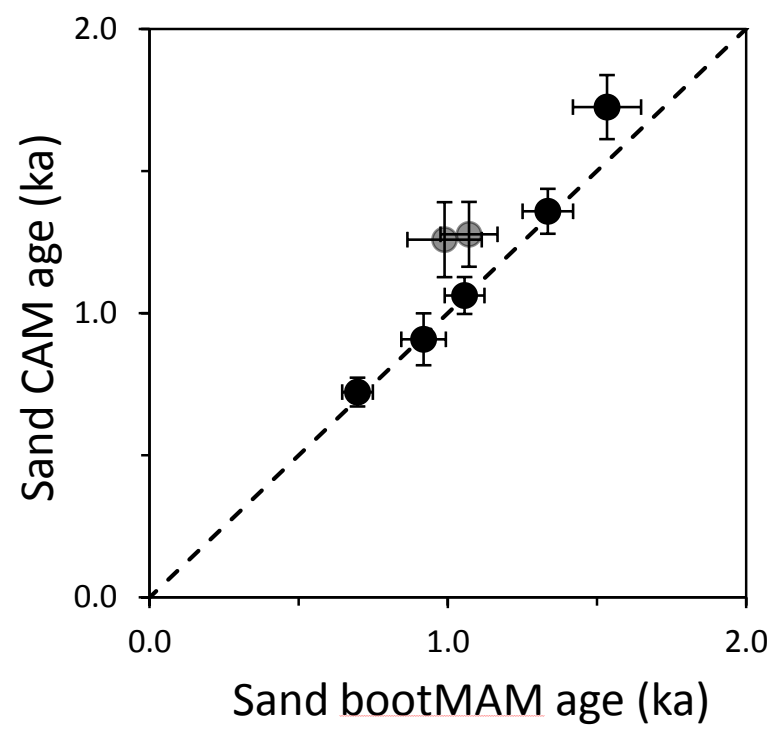

Figure S3. Comparison of sand ages obtained with CAM and bootMAM for 7 samples used in the paired sand/silt analysis. Gray circles indicate PV I-4 and PV I-5, two samples possibly affected by feldspar contamination or containing poorly bleached silt.

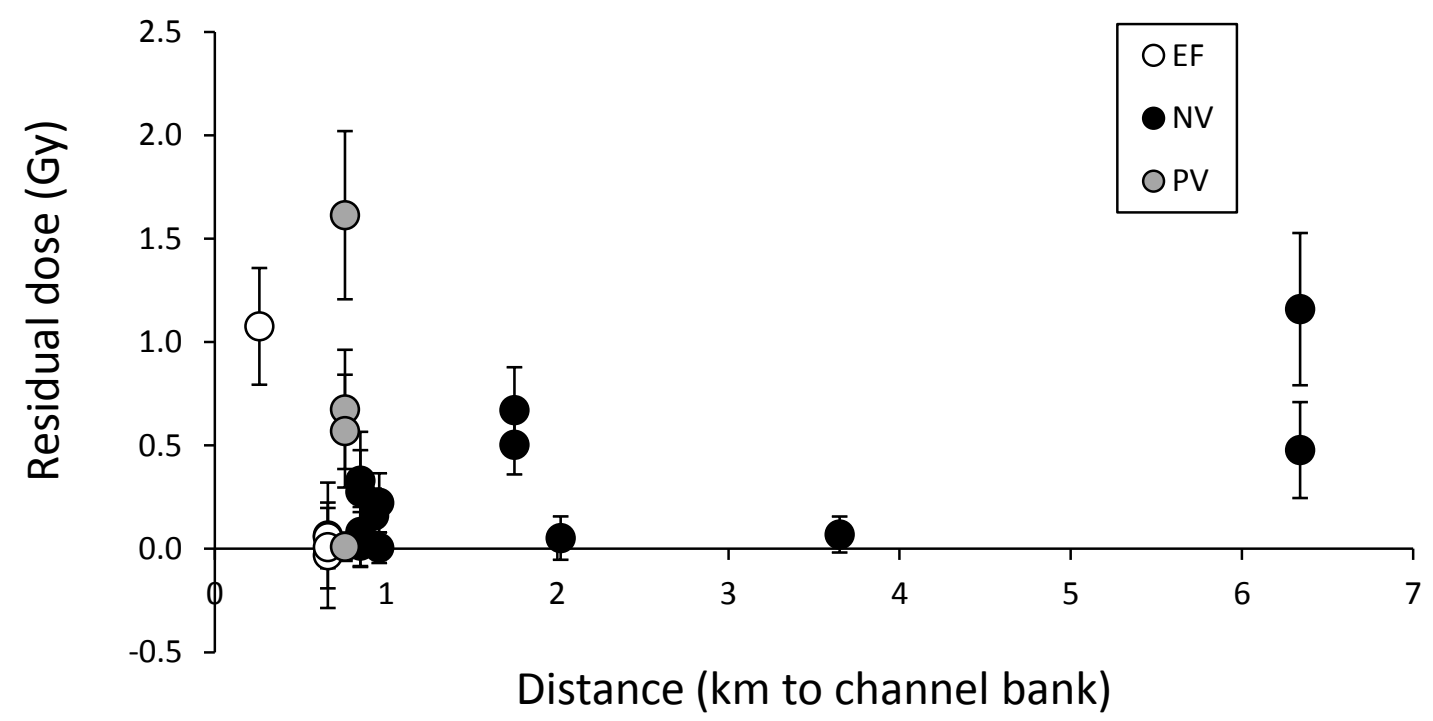

Figure S4. No spatial trend in bleaching was observed in overbank sands sampled at the Elmfield (EF), Napoleonville (NV) and Paincourtville (PV) sites, relative to the channel bank of Bayou Lafourche. 


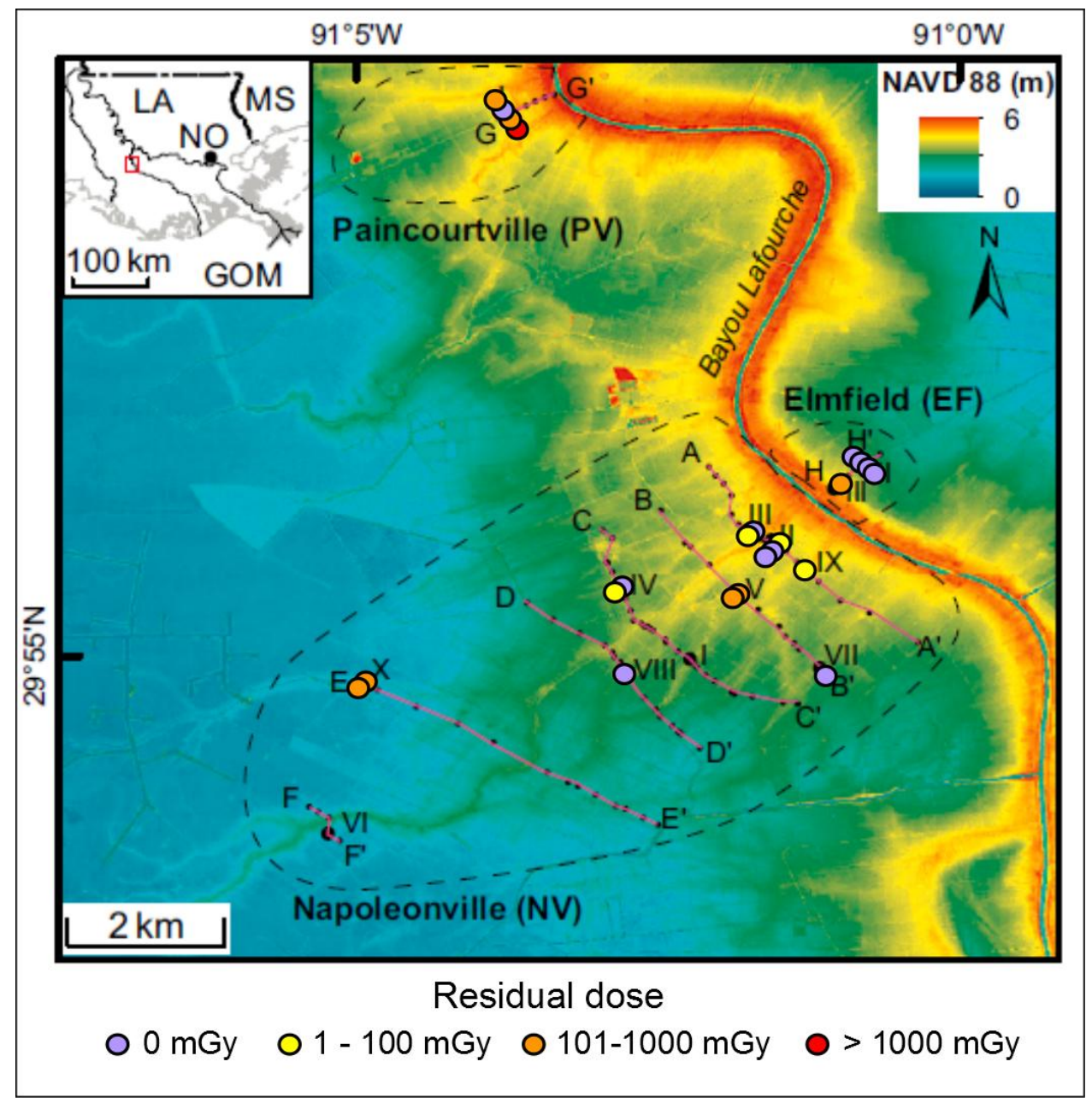

Figure S5. Details of the spatial distribution of overbank sand residual doses. The base map is modified from Shen et al. (2015). Samples from within the same borehole are indicated by stacked (overlapping) symbols, with the deepest sample at the base of the stack (most obscured symbol) and the shallowest sample at the top (most visible symbol). The orientation of the stacks is not meaningful. 